\title{
Efeitos da História Comportamental e de Instruções sobre a Aquisição e a Resistência à Extinção em um Esquema Múltiplo FR DRL
}

\author{
Celso Apparecido Athayde Neto \\ Centro Universitário Filadélfia \\ Carlos Eduardo Costa \\ Universidade Estadual de Londrina \\ Roberto Alves Banaco \\ Pontifícia Universidade Católica de São Paulo
}

\begin{abstract}
RESUMO - Para verificar os efeitos da história comportamental e de instruções sobre a aquisição e a resistência à extinção, 15 universitários foram distribuídos em três grupos. Na Fase 1, o Grupo 1 foi exposto a aumentos graduais nos valores dos componentes até um múltiplo FR 60 DRL 20 s; os grupos 2 e 3 foram expostos diretamente a esse esquema, mas o Grupo 3 recebeu instruções. Na Fase 2, o FR foi ajustado e um múltiplo FR n DRL 20 s foi mantido até a estabilidade. Na Fase 3 estava em vigor um múltiplo Extinção Extinção. As instruções, em comparação com a exposição gradual ou direta aos esquemas, facilitaram a aquisição de taxas diferenciadas de respostas, mas aumentaram a resistência à extinção.
\end{abstract}

Palavras-chave: história comportamental, instruções, esquemas múltiplos, aquisição, resistência à mudança, humanos

\section{The Effects of Behavioral History and Instructions upon the Acquisition and Resistance to Extinction with a Multiple FR DRL Schedule}

\begin{abstract}
To investigate the effects of the behavioral history and instructions on acquisition and resistance to extinction with a multiple schedule, 15 students were distributed into three groups. In Phase 1, Group 1 was exposed to gradual increases in the values of the components until a multiple FR 60 DRL $20 \mathrm{~s}$; groups 2 and 3 were directly exposed to that multiple schedule, but Group 3 received instructions. In Phase 2, the FR was adjusted and a multiple FR n DRL $20 \mathrm{~s}$ was maintained until stability. In Phase 3, a multiple Extinction schedule was in effect. Instructions, as compared to direct or gradual exposure to the schedules, facilitated acquisition of differentiated response rates, but increased resistance to extinction.
\end{abstract}

Keywords: behavioral history, instructions, multiple schedules, acquisition, resistance to extinction, humans

A diferenciação na taxa de respostas em um esquema múltiplo razão fixa (FR) reforçamento diferencial de taxas baixas (DRL) com humanos - taxas de respostas altas no FR e baixas no DRL - nem sempre é observada: taxas de respostas diferenciadas ocorreram, em alguns estudos, para a maioria (e.g., Okouchi, 1999) ou para todos os participantes (e.g., Costa, Soares, \& Ramos, 2012; Porto, Ramos, \& Costa, 2011; Rumbold \& White, 1987; Soares, Costa, Cançado, \& Cirino, 2013), enquanto outros estudos relataram dificuldades em se obter tal diferenciação, a menos que instruções sobre como responder em cada componente do múltiplo fosse fornecida (e.g., Hayes, Brownstein, Hass, \& Greenway, 1986; Hayes, Brownstein, Zettle, Rosenfarb, \& Korn, 1986, Experimento 1; Rosenfarb, Newland, Brannon, \& Howey, 1992).

Uma diferença relevante entre os estudos que obtiveram ou não diferenciação nas taxas de respostas diz respeito à história de exposição aos parâmetros finais do múltiplo FR DRL: exposição direta (e.g., Hayes, Brownstein, Hass et al., 1986; Hayes, Brownstein, Zettle et al., 1986; Rosenfarb et al., 1992) em contraposição à exposição gradual a esses parâmetros (e.g., Costa et al., 2012; Okouchi, 1999; Porto

1 Instituto de Psicologia e Análise do Comportamento, IPAC, Rua Pará, 1753, Centro, Londrina, PR, Brasil. CEP: 86020-400. E-mail: netoathayde@gmail.com et al., 2011; Rumbold \& White, 1987). Taxas de respostas diferenciadas são mais comumente observadas com mudança gradual, intra ou entre sessões, nos parâmetros. Por exemplo, Porto et al. (2011) distribuíram universitários em quatro grupos com diferentes histórias de aquisição em um múltiplo FR 60 DRL 20 s: Grupo 1-Incremento intrassessão e não simultâneo do FR e do DRL (aumento primeiro no parâmetro do DRL e depois no do FR); Grupo 2-Incremento intrassessão e simultâneo do FR e do DRL; Grupo 3-Incremento do FR e do DRL entre sessões; e Grupo 4-Incremento do FR e do DRL entre sessões. Para os Grupos 1, 2 e 3, o critério para mudança de componente (FR para DRL e vice-versa) foi a obtenção de 10 pontos e para o Grupo 4, o critério para mudança de componente era de tempo fixo (3 minutos). Os resultados indicaram que houve diferenciação na taxa de respostas para os quatro grupos e não houve diferença no número de sessões para a obtenção de taxa de respostas diferenciadas. Aparentemente, tanto a forma do aumento gradual nos parâmetros do FR e do DRL, quanto o critério para alternância dos componentes (pela passagem de um período de tempo fixo vs. pela obtenção de certo número de pontos) podem produzir taxa de respostas diferenciadas entre os componentes. 
A diferenciação na taxa de respostas em um esquema múltiplo FR DRL também pode ser obtida se uma instrução sobre como responder em cada esquema for fornecida ao participante (e.g., Hayes, Brownstein, Hass, et al., 1986; Hayes, Brownstein, Zettle, et al., 1986, Experimento 1; Rosenfarb et al., 1992). Todavia, apesar da aquisição de novos comportamentos ser facilitada pelo uso de instruções (e.g., Catania, 1999; Cerutti, 1989; Skinner, 1969), os resultados de algumas pesquisas (e.g., Albuquerque, Souza, Matos, \& Paracampo, 2003; Hayes, Brownstein, Haas et al., 1986; Rosenfarb et al., 1992) sugerem também que instruções podem reduzir a sensibilidade (ou, alternativamente, aumentar a resistência) comportamental às mudanças nas contingências de reforço programadas ${ }^{1}$. Por exemplo, no estudo de Hayes, Brownstein, Haas et al. (1986), os participantes do Grupo $1(n=19)$ foram expostos diretamente ao esquema múltiplo FR DRL, recebendo apenas instruções mínimas sobre a tarefa, e os do Grupo $4(n=16)$ receberam a instrução de responder rápido na presença do FR e devagar na presença do DRL (i.e., instruções correspondentes). Em seguida, todos os participantes foram expostos ao múltiplo Extinção Extinção.

Os resultados indicaram que apenas seis participantes do Grupo 1 emitiram um responder diferenciado na primeira fase, mas todos esses seis apresentaram redução na taxa de respostas durante a extinção; por outro lado, dos 15 participantes do Grupo 4 que emitiram taxas de respostas diferenciadas, apenas oito reduziram a taxa de respostas. Os autores concluíram que as instruções facilitaram a aquisição de taxas diferenciadas, mas aumentaram a resistência dessas taxas à retirada do reforço.

Em suma, a literatura sugere que a história de exposição ao esquema múltiplo FR DRL (direta vs. gradual) afeta a aquisição de taxas diferenciadas de respostas nesses componentes. No entanto, essa sugestão resulta da comparação entre estudos que diferiram não somente porque usaram ou exposição direta ou exposição gradual, mas também por outros aspectos metodológicos, o que dificulta afirmações inequívocas sobre o papel da exposição direta ou gradual. Dessa forma, um dos objetivos do presente estudo consistiu em isolar o efeito dessa variável. Para tanto, dois grupos de participantes foram expostos a contingências que se diferenciaram apenas em termos da história de exposição ao múltiplo FR DRL.

A literatura também indica que instruções aumentam a resistência do comportamento a mudanças. Como base nisso, outro objetivo do presente estudo foi avaliar se a resistência à extinção seria afetada não somente pela presença $v s$. ausência de instruções, mas também pela história de exposição (direta vs. gradual) ao múltiplo FR DRL. Para alcançar esse objetivo,

1 No presente estudo, as expressões "(in)sensibilidade comportamental" e "resistência do comportamento à mudança" foram usadas de modo intercambiável, apesar da origem de cada uma assentar-se em diferentes temas de pesquisa (i.e., comportamento governado por regras e momentum comportamental, respectivamente) com diferentes procedimentos. A semelhança entre esses temas de pesquisa foi apontado por dos Santos (2005). No presente trabalho, as expressões referem-se ao grau de mudança replicável e sistemática de um comportamento frente à(s) mudança(s) nas contingências (cf. Madden, Chase, \& Joyce, 1998). um terceiro grupo de participantes recebeu instruções correspondentes a esse esquema.

\section{Método}

O presente trabalho foi aprovado (Parecer 108/10) pelo Comitê de Ética em Pesquisa Envolvendo Seres Humanos da Universidade Estadual de Londrina (Registro CONEP 268).

\section{Participantes}

Participaram 15 estudantes universitários, de ambos os sexos (11 mulheres e quatro homens), com faixa etária entre 20 e 30 anos. Foram excluídos estudantes que cursavam Psicologia ou tinham conhecimento sobre esquemas de reforço, bem como aqueles com diagnósticos ou queixa de LER (Lesão por Esforço Repetitivo) ou DORT (Distúrbio Osteomuscular Relacionado ao Trabalho).

\section{Local, Equipamentos e Instrumentos}

Três salas de, aproximadamente, $3 \mathrm{~m}^{2}$ cada, foram usadas. Em cada sala experimental havia uma mesa, uma cadeira e um computador do tipo PC, com monitor em cores de 14 polegadas, mouse e teclado padrões, fone de ouvido e filmadora digital com tripé. O instrumento utilizado para a coleta de dados foi o software ProgRef v3.1 (Costa \& Banaco, 2002, 2003) e para o cálculo de estabilidade da taxa de respostas, o software Stability Check (Costa \& Cançado, 2012).

\section{Procedimento}

Antes da primeira sessão, os participantes receberam o Termo de Consentimento Livre e Esclarecido (TCLE). O TCLE informava que o objetivo da pesquisa era "estudar algumas variáveis que possam afetar o modo como as pessoas se comportam em determinadas situações", que o participante tinha que "ganhar o maior número de pontos possíveis (que aparecerão na tela do monitor) utilizando o mouse", que as sessões durariam 30 minutos cada e que os pontos ganhos seriam trocados por dinheiro $(\mathrm{R} \$ 0,05)$ ao final de cada sessão.

Cada participante era instruído a deixar todo material, incluindo celular e relógio, no laboratório, fora da sala experimental, e só então era convidado a entrar na sala experimental, sentar-se e ler as seguintes instruções escritas:

Esse estudo não consiste em uma pesquisa sobre inteligência ou personalidade. Seu objetivo será ganhar pontos utilizando apenas o mouse [Para ganhar tantos pontos quanto possivel, você deverá pressionar rápido quando o botão estiver Azul e devagar quando o botão estiver Amarelo]. Os pontos aparecerão em uma janela (contador) que se localizará na parte superior da tela do computador na posição central. O experimentador não está autorizado a dar qualquer informação adicional. Caso haja dúvidas, releia o texto acima e prossiga o experimento. Bom trabalho! 
A parte da instrução contida dentro dos colchetes era exibida apenas para os participantes do Grupo 3 da pesquisa.

Para que ruídos de fora da sala experimental não interferissem no desempenho do participante, era solicitado a ele que utilizasse o fone de ouvido, através do qual soava um ruído branco. Houve no máximo duas sessões por dia, entre as $7 \mathrm{~h}$ e $20 \mathrm{~h}$, de segunda à sexta-feira, exceto feriados. Os participantes foram distribuídos em três grupos, conforme Tabela 1.

Tabela 1. Resumo do procedimento experimental

\begin{tabular}{lcccc}
\hline \multirow{2}{*}{ Fases } & $\begin{array}{c}\text { Grupos } \\
(\mathbf{n = 5 )}\end{array}$ & Sessão & \multicolumn{2}{c}{$\begin{array}{c}\text { Componentes dos } \\
\text { esquemas múltiplos }\end{array}$} \\
\cline { 3 - 5 } & & Sessão 1 & FR 15 & DRL 5 s \\
& Grupo 1 & Sessão 2 & FR 30 & DRL 10 s \\
Fase 1 & & Sessão 3 & FR 60 & DRL 20 s \\
& Grupo 2 & Sessão 1 & FR 60 & DRL 20 s \\
& Grupo 3 & Sessão 1 & FR 60 & DRL 20 s \\
Fase 2 & Grupos 1, 2 e 3 & ------- & FR $n^{\text {a }}$ & DRL 20 s \\
Fase 3 & Grupos 1, 2 e 3 & ------- & Extinção & Extinção \\
\hline
\end{tabular}

Nota: a $\mathrm{O}$ valor da razão foi ajustado para cada participante, para que o tempo para a emissão do número de respostas necessárias para a obtenção de pontos fosse de, aproximadamente, $20 \mathrm{~s}$

Tarefa experimental. A tela do computador consistia em um fundo cinza e continha um botão na posição central superior com a palavra "Iniciar". Ao clicar nesse botão, a sessão era iniciada com a apresentação de outra tela em um fundo cinza, contendo um retângulo no centro inferior (botão de respostas), um contador de pontos no centro superior e um retângulo no canto superior direito (botão de resposta de consumação). Considerou-se uma resposta, cada clique no botão esquerdo do mouse com o cursor sobre o botão de respostas. Quando a exigência do esquema de reforço era cumprida, aparecia um smile (ícone de expressão facial sorridente) abaixo do botão de resposta de consumação.

Um clique sobre o botão de resposta de consumação, enquanto o smile estava presente, produzia o acúmulo de um ponto no contador e o desaparecimento do smile. Durante a vigência de um DRL, o início do intervalo se dava com o aparecimento do smile na tela. Dessa forma, o tempo gasto pelo participante em migrar o cursor do mouse do botão de respostas ao botão da resposta de consumação, clicar no botão de consumação e finalmente retornar o cursor ao botão de respostas era computado como parte do intervalo do DRL. Respostas emitidas no botão de respostas quando o smile estivesse presente na tela eram registradas, mas outro smile não aparecia até que a resposta de consumação fosse emitida (i.e., não havia um limted hold programado). Cada componente do esquema múltiplo durava $3 \mathrm{~min}$ e era apresentado cinco vezes, em alternação simples. Nas Fases 1 e 2, os parâmetros dos componentes sempre reiniciavam cada vez que o componente entrava em vigor. Por exemplo, mesmo que o participante, ao terminar os 3 min do componente FR 60, tivesse emitido 30 respostas, necessitaria emitir 60 respostas no próximo FR para obter um ponto. A mesma estratégia foi aplicada ao componente DRL. Esse procedimento de alternação foi usado com o objetivo de replicar o procedimento utilizado por Porto et al. (2011) e, assim, facilitar comparação entre estudos.

Fase 1 - Aquisição. Durante essa fase, um múltiplo FRDRL estava em vigor. No Componente FR, a cor do botão de respostas era azul, e durante o Componente DRL, amarelo. Havia um intervalo entre componentes (IEC) que durava $5 \mathrm{~s}$, com a tela do computador escura e a palavra "Aguarde", em letras vermelhas, exibida no centro da tela. Os botões de resposta e de consumação e o contador de pontos desapareciam durante o IEC e qualquer clique no mouse não tinha efeito.

Para os participantes do Grupo 1-Exposição gradual, houve incremento gradual dos valores de ambos os componentes do esquema múltiplo, entre sessões: Na primeira, segunda e terceira sessão, os valores dos componentes eram FR 15 e DRL 5 s; FR 30 e DRL 10 s; e FR 60 e DRL 20 s (esse procedimento foi semelhante ao utilizado para o Grupo 4 de Porto et al., 2011). Os participantes do Grupo 2-Exposição direta e do Grupo 3-Exposição direta com instrução foram submetidos, desde o início do experimento, a um múltiplo FR 60 DRL 20 s. A diferença entre o Grupo 2 e 3 estava na instrução. Os participantes do Grupo 2 receberam a mesma instrução dada ao Grupo 1, e os participantes do Grupo 3 receberam essa instrução com a adição do texto entre colchetes.

A Fase 1 era finalizada quando dois critérios eram atingidos na mesma sessão: (1) índice de diferenciação (ID) na taxa de respostas $\geq 0,9$ e (2) acúmulo de pelo menos $50 \%$ dos pontos disponíveis no componente DRL (para garantir que o comportamento havia feito contato com a contingência de reforço diversas vezes). O ID foi calculado pela divisão do número total de respostas emitidas no FR pela soma do número de respostas emitidas no FR e no DRL (cf. Rosenfarb, et al., 1992). O resultado variava de 0 a 1, sendo que 0,5 indicava que a taxa de respostas era igual no FR e no DRL; 0,0 representava taxas de respostas totalmente diferenciadas, mas sem emissão de respostas no FR; e 1,0 representava taxa de respostas totalmente diferenciada, mas com nenhuma resposta emitida no DRL. Caso algum destes critérios não fosse alcançado, era programada uma nova sessão com as mesmas características. Assim como em Porto et al. (2011), esses critérios começaram a ser considerados a partir da segunda sessão para os participantes do Grupo 1, pois o valor dos componentes na primeira sessão não exigia grande diferenciação da taxa de respostas, o que dificultava o alcance do critério de diferenciação da taxa de respostas. Os participantes da pesquisa que não atingiram ambos os critérios em até quatro exposições aos mesmos valores de esquema de reforços foram excluídos da pesquisa.

Fase 2 - Linha de base. Nessa fase era realizada a aproximação dos intervalos entre reforços (IRIs) dos componentes do esquema múltiplo e, subsequentemente, era avaliada a estabilidade da taxa de respostas. Para aproximação dos IRIs, o valor do FR foi alterado com base na média do IRI no DRL da sessão imediatamente anterior (um procedimento semelhante foi utilizado por Soares et al., 2013). Por exemplo, se o IRI médio do DRL durante uma sessão fosse igual a $22 \mathrm{~s}$, o participante ganharia, aproximadamente, 40 pontos nos 15 min desse componente. Se, na mesma sessão, nos 15 min de exposição ao FR 60 o participante ganhasse 60 pontos, isso 
indicaria que o IRI médio no FR foi de, aproximadamente, 15 s. Então, uma regra de três era aplicada (se 60 respostas foram emitidas em $15 \mathrm{~s}$, quantas respostas seriam emitidas em 22 s?). Na sessão seguinte, então, seria programado um FR 88: com esse valor, se o participante mantivesse a mesma taxa de respostas ele obteria, aproximadamente, 40 pontos em $15 \mathrm{~min}$, o que significaria um ponto a cada $22 \mathrm{~s}$. Esse procedimento foi realizado para evitar que a taxa de pontos interferisse na avaliação da resistência à mudança (cf. Nevin, 1974). As taxas de pontos foram consideradas aproximadas quando a diferença entre os pontos ganhos em cada esquema era menor ou igual a 15 pontos totais na sessão.

Uma vez determinado o valor do FR (n) que produzia um IRI semelhante ao IRI do DRL, os participantes eram expostos ao múltiplo FR n DRL $20 \mathrm{~s}$, até que as taxas de respostas fossem estáveis. Para avaliar a estabilidade da taxa em FR foi utilizado um critério relativo (cf. Schoenfeld, Cumming, \& Hearst, 1956), segundo o qual a diferença entre a média da taxa de respostas das duas primeiras sessões e a média da taxa das duas últimas sessões do bloco composto pelas quatro últimas sessões realizadas, não podiam variar mais do que $10 \%$. Para a taxa DRL foi utilizado um critério absoluto, ou seja, a taxa era considerada estável quando não variava em mais do que três respostas por minuto, para mais ou para menos, nas últimas quatro sessões. Foi utilizado um critério de estabilidade diferente para a taxa DRL porque, para taxas de respostas baixas, qualquer variação mínima na taxa absoluta pode representar uma grande variação na taxa relativa de respostas (cf. Perone, 1991; Porto et al., 2011).

Fase 3 - Teste. Na sessão seguinte à obtenção do critério de estabilidade, ocorria a suspensão da liberação de pontos no programa múltiplo, ou seja, um múltiplo Extinção Extinção entrava em vigor. A duração dos componentes, a alternação entre eles, o IEC e as cores dos botões nos quais operavam os esquemas de reforço permaneceram inalterados. Cinco sessões de 30 min cada foram realizadas para avaliar a resistência do comportamento à mudança (cf. Nevin, 1974). Nessa fase, o componente de extinção, sinalizado pela cor correlacionada com o FR das fases anteriores, foi denominado de "FR", e o componente sinalizado pela cor correlacionada com o DRL das fases anteriores foi denominado de "DRL".

O participante que não emitiu nenhuma resposta em duas apresentações seguidas do "FR" e do "DRL" teve a participação na pesquisa encerrada, pois foi admitido que, nessas condições, a taxa de respostas havia extinguido.

\section{Resultados}

A Figura 1 exibe a taxa de respostas por minuto (R/ min) em cada sessão para cada participante e a Tabela 2 exibe o índice de diferenciação (ID) e a porcentagem de pontos ganhos no DRL (\%DRL) na Fase 1-Aquisição (parte à esquerda do gráfico de cada participante na Figura 1). Verifica-se que, dentre os participantes do Grupo 1 (Exposição gradual), P1 e P4 atingiram o esquema múltiplo FR 60 DRL 20 s após uma única exposição a cada um dos esquemas múltiplos planejados no procedimento (ver Tabela 1), totalizando três sessões nessa fase (o mínimo possível); P2, P3 e P5, por outro lado, foram expostos a uma sessão no múltiplo FR 15 DRL 5 s e a duas sessões do múltiplo FR 30 DRL $10 \mathrm{~s}$ antes de serem expostos ao múltiplo FR 60 DRL $20 \mathrm{~s}$, perfazendo um total de quatro sessões na Fase 1.O ID final dos participantes desse grupo foi igual ou superior a 0,98 e a porcentagem de pontos obtidos em DRL foi superior a 60 para quatro dos cinco participantes.

Dos participantes que foram expostos diretamente ao múltiplo FR 60 DRL 20 s, sem instrução acerca da taxa de respostas (Grupo 2-Exposição direta), P7 atingiu ambos os critérios de aquisição do comportamento em duas sessões (com ID igual a 0,97 e porcentagem de pontos ganhos em DRL igual a 52,5), e P6 e P10, em quatro (com o ID de 0,9 e a porcentagem de pontos ganhos em DRL acima de 70). Os participantes P8 e P18 não atingiram os critérios de aquisição do comportamento após quatro sessões, sendo dispensados da pesquisa. O participante P8 emitiu taxas de respostas suficientemente diferenciadas logo na segunda sessão, mas não obteve nenhum ponto em DRL nas quatro sessões. $\mathrm{O}$ participante P18, por sua vez, apresentou um aumento gradativo no ID, atingindo o valor de 0,89 e obtenção de $42 \%$ dos pontos disponíveis em DRL na quarta sessão. Os participantes do Grupo 3 (Exposição direta com instrução) necessitaram de duas (P11 e P15) a três sessões (P12 e P16) para atingirem os critérios de aquisição do comportamento, com ID igual ou superior a 0,92 e porcentagem de pontos obtidos em DRL igual ou acima de 60 para três dos quatro participantes. O participante $\mathrm{P} 13$ emitiu taxas de respostas diferenciadas, mas não obteve pontos no DRL, sendo dispensado da pesquisa após a quarta sessão dessa fase.

Esses resultados indicam que, o Grupo 1 foi o único em que os cinco participantes atingiram os critérios de aquisição do comportamento na Fase 1, o que indica que a diferenciação entre taxas FR e DRL é mais provável (ou consistente) quando há exposição gradual ao esquema múltiplo. Os resultados mostram também que as instruções sobre como responder em cada componente do múltiplo (Grupo 3) aceleram a aquisição de taxas diferenciadas, em comparação à exposição abrupta ao esquema, sem instruções (Grupo 2). Isto porque, no Grupo 2, três dos cinco participantes (P6, P7 e $\mathrm{P} 10)$ atingiram os critérios e dois deles precisaram de quatro sessões para isso, enquanto no Grupo 3, quatro de cinco participantes (P11, P12, P15 e P16) atingiram os critérios e precisaram de duas a três sessões para isso. Essas diferenças entre grupos, entretanto, devem ser vistas com cautela, uma vez que, em termos do número de sessões para atingir ambos os critérios e dos valores finais obtidos para ambos os critérios (ID e porcentagem de pontos ganhos no DRL), a diferença entre grupos foi pequena e houve considerável variabilidade dentro dos grupos.

Durante a Fase 2-Linha de Base, o valor do FR foi alterado com o objetivo de aproximar os valores do IRI entre componentes. Os valores finais do múltiplo FR DRL bem como a taxa de pontos (pontos/min) obtidos em cada componente, considerando apenas a última sessão dessa fase e considerando toda a Fase 1 e a Fase 2, podem ser observados na Tabela 3. Observa-se que os valores do FR foram estabelecidos entre 100 e 135 respostas. A diferença na taxa de pontos ganhos entre os dois componentes na última sessão da Fase 2 não ultrapassou 1 ponto/min. A média da taxa de pontos obtida em cada componente, para todos os 
participantes, foi de 2,38 pontos/min no FR e 1,96 pontos/min no DRL. Porém, quando se toma em consideração para os cálculos todas as sessões das Fases 1 e 2, a diferença na taxa média de pontos obtidos entre os componentes aumenta, já que na Fase 1 o valor do FR era menor do que foi na Fase 2.
Foram necessárias de quatro a oito sessões, após a Fase 1, para que o IRI entre componentes apresentasse valores aproximados (dados não exibidos) e a estabilidade da taxa de respostas fosse atingida por todos os participantes. Houve um erro de programação na oitava sessão do experimento

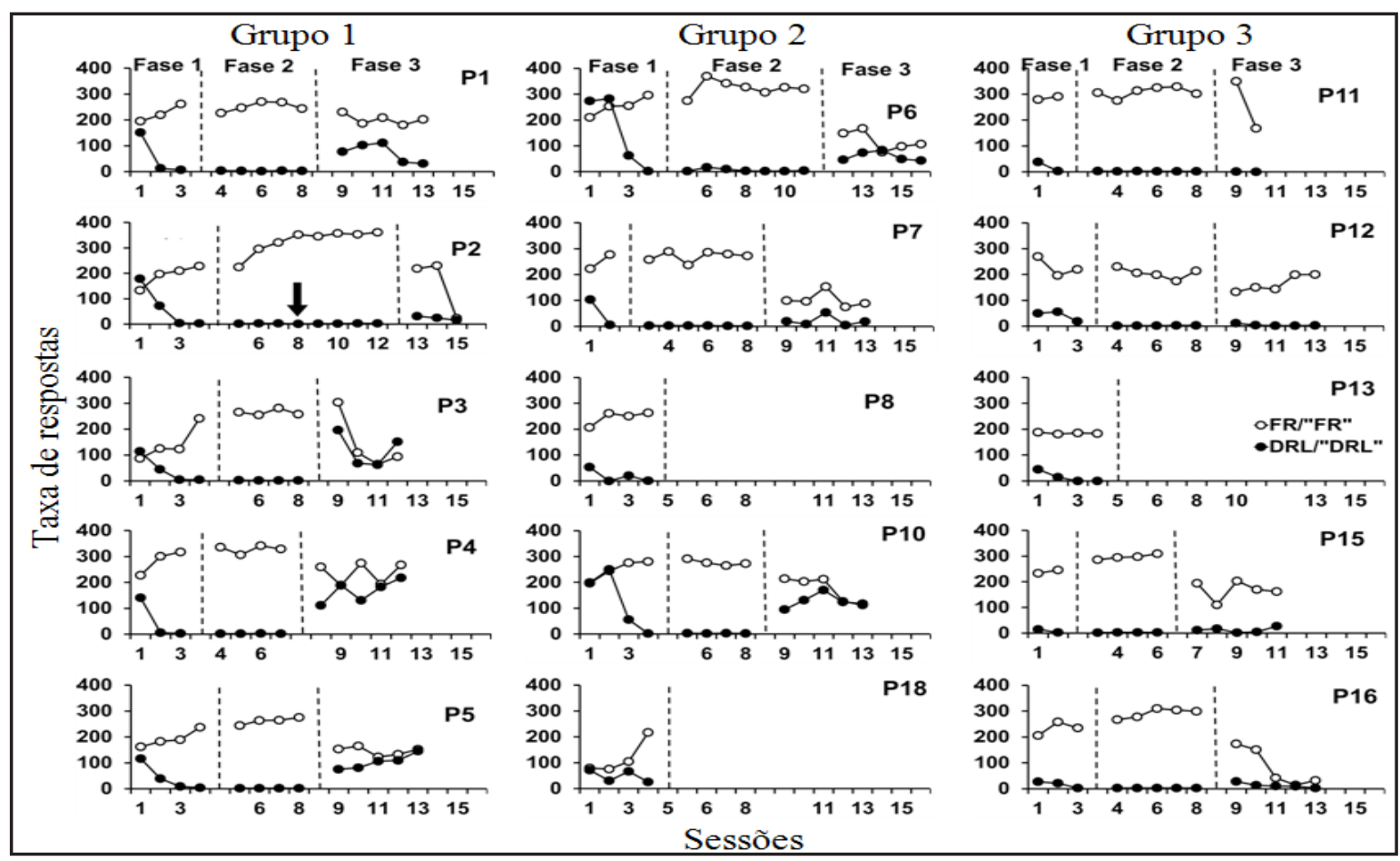

Figura 1. Taxa de respostas por minuto em cada sessão de cada participante. O círculo vazio identifica a taxa de respostas no FR durante as fases 1 e 2 e "FR" na Fase 3; o círculo cheio identifica a taxa de respostas no DRL durante as fases 1 e 2 e "DRL" na Fase 3 para o Grupo 1-Exposição gradual, Grupo 2-Exposição direta e Grupo 3-Exposição direta com instrução. Os traços verticais pontilhados separam as Fases 1, 2 e 3, da esquerda para direita.

Tabela 2. Índice de diferenciação (ID) da taxa de respostas e porcentagem de pontos ganhos no DRL em relação aos pontos disponíveis (\%DRL) na Fase 1-Aquisição

\begin{tabular}{|c|c|c|c|c|c|c|c|c|c|}
\hline \multirow{2}{*}{ Grupo } & \multirow{2}{*}{ Participantes } & \multicolumn{2}{|c|}{ Primeira Sessão } & \multicolumn{2}{|c|}{ Segunda Sessão } & \multicolumn{2}{|c|}{ Terceira Sessão } & \multicolumn{2}{|c|}{ Quarta Sessão } \\
\hline & & ID & $\% \mathrm{DRL}$ & ID & $\% \mathrm{DRL}$ & ID & $\% \mathrm{DRL}$ & ID & $\% \mathrm{DRL}$ \\
\hline \multirow{5}{*}{ G1 } & $\mathrm{P} 1$ & 0,56 & 19,4 & 0,94 & 61,1 & 0,97 & 50,0 & --- & --- \\
\hline & $\mathrm{P} 2 *$ & 0,42 & 1,1 & 0,73 & 8,0 & 0,98 & 58,8 & 0,98 & 70,0 \\
\hline & $\mathrm{P} 3 *$ & 0,43 & 3,4 & 0,73 & 43,0 & 0,96 & 70,5 & 0,98 & 67,5 \\
\hline & P4 & 0,61 & 32,5 & 0,98 & 78,0 & 0,99 & 80,0 & --- & --- \\
\hline & $\mathrm{P} 5 *$ & 0,58 & 20,0 & 0,82 & 36,4 & 0,95 & 71,7 & 0,98 & 62,5 \\
\hline \multirow{5}{*}{ G2 } & P6 & 0,43 & 0,0 & 0,47 & 0,0 & 0,80 & 45,0 & 0,99 & 77,5 \\
\hline & P7 & 0,68 & 12,5 & 0,97 & 52,5 & --- & --- & --- & --- \\
\hline & P8 & 0,79 & 0,0 & 1 & 0,0 & 0,92 & 0,0 & 0,99 & 0,0 \\
\hline & P10 & 0,49 & 2,5 & 0,49 & 0,0 & 0,83 & 47,5 & 0,99 & 72,5 \\
\hline & P18 & 0,52 & 2,5 & 0,71 & 2,5 & 0,61 & 0,0 & 0,89 & 42,0 \\
\hline \multirow{5}{*}{ G3 } & P11 & 0,88 & 0,0 & 0,99 & 57,5 & --- & --- & --- & --- \\
\hline & P12 & 0,84 & 0,0 & 0,77 & 0,0 & 0,92 & 60,0 & --- & --- \\
\hline & P13 & 0,80 & 0,0 & 0,92 & 0,0 & 1 & 0,0 & 1 & 0,0 \\
\hline & P15 & 0,94 & 25,0 & 0,98 & 82,5 & --- & --- & --- & --- \\
\hline & P16 & 0,88 & 0,0 & 0,92 & 30,0 & 0,98 & 72,5 & --- & --- \\
\hline
\end{tabular}


(sinalizado por uma seta na Figura 1) do participante P2 do Grupo 1, uma vez que o valor do DRL foi de $50 \mathrm{~s}$ (em vez de $20 \mathrm{~s}$ ) e, por isso, a estabilidade foi calculada utilizando-se as quatro sessões posteriores a essa sessão.

Conforme observado na Figura 1, a tendência geral das taxas de respostas dos participantes quando a contingência de reforço mudou para múltiplo EXT EXT (parte à direita do gráfico de cada participante na Figura 1), mantendo os estímulos discriminativos das fases anteriores, é possível notar diminuição na taxa de respostas durante o "FR" e aumento na taxa de respostas durante o "DRL" para a maioria dos participantes dos três grupos. Apenas P1 (Grupo 1) manteve taxas de respostas relativamente altas no "FR" em todas as sessões de extinção (acima de $181 \mathrm{R} / \mathrm{min}$ ). Pode-se notar, também, que P3 (Grupo 1) e P11 (Grupo 3) mantiveram taxas de respostas relativamente altas no "FR", mas apenas na primeira sessão de extinção (303 e 349 R/ min, respectivamente).

A Figura 2 apresenta a proporção de mudança da taxa de respostas (em escala logarítmica) na Fase 3-Teste, em relação à Fase 2-Linha de base (cf. Nevin, 1974, 1979). Essa medida foi calculada dividindo-se a taxa de respostas do componente "FR" ("DRL") em cada sessão da Fase 3 pela taxa de respostas no componente FR (DRL) da última sessão da linha de base (Fase 2), sendo os resultados expressos em escala logarítmica no eixo y. Quanto mais próximo o valor obtido for de 1,0, menor a mudança da taxa de respostas em extinção em relação à Linha de Base, ou seja, maior a resistência à extinção. As linhas tracejadas em 10,0 e 0,1 indicam uma mudança de 10 vezes o valor da linha de base, para mais ou para menos, respectivamente. Os gráficos superiores exibem a média dos participantes de cada grupo.

Pode-se observar que, de forma geral, a taxa de respostas no "FR" diminuiu, enquanto que a taxa de respostas no "DRL" aumentou em relação à LB. Apenas P11 teve diminuição na taxa de respostas, em relação à $\mathrm{LB}$, em ambos os componentes. Para cinco (P1, P3, P4, P5 e P10) dos oito participantes que não receberam instruções (Grupos 1 e 2), a taxa no "DRL" foi 10 vezes maior do que a taxa de respostas no componente DRL durante a LB, enquanto essa magnitude de mudança na taxa de respostas não foi observada para nenhum dos participantes que obtiveram instruções (Grupo 3). Ou seja, houve maior resistência à mudança no "DRL" quando instruções foram usadas. Quando comparados os componentes "FR" e "DRL", observa-se maior resistência à mudança na presença do "FR".

Embora a proporção de mudança das taxas de respostas do "FR" das sessões de extinção não indique diferenças entre grupos (Figura 2), os registros cumulativos exibidos na Figura 3 , a seguir, tornam possível a identificação do momento em que houve alteração no padrão de respostas no "FR", que foi sempre o primeiro componente da Extinção para todos os participantes.

Para todos os participantes, foram observadas taxas de respostas relativamente altas e sem pausa durante a última apresentação do FR e taxas de respostas relativamente mais baixas durante a última apresentação do DRL da Fase 2. Após a suspensão do reforço, com manutenção dos estímulos anteriormente correlacionados com FR e DRL (Fase 3), P1, P2, P3, P4, P6 e P7 fizeram pausa no responder, identificada pelas letras a, durante a primeira apresentação do "FR" da Fase 3, e essa pausa ocorreu no segundo minuto de extinção para P1, P2, P6 e P7. A ocorrência de pausa durante o "FR" não foi notada entre os participantes do Grupo 3 nos três minutos iniciais do "FR", mas P16, apresentou uma diminuição da taxa de respostas nesse período (apontada pela letra b). Outro aspecto que pode ser observado na Figura 3 é que, enquanto na primeira exposição ao "DRL" há jorros (bursts) de respostas (apontados pela letra c) para os oito

Tabela 3. Valor final do Componentel (FR) do esquema múltiplo $F R \neg D R L$, taxa de pontos ganhos (pontos/min) em cada componente na última sessão da Fase 2 e taxa de pontos (pontos/min) nas Fases 1 e 2

\begin{tabular}{|c|c|c|c|c|c|}
\hline \multirow[b]{2}{*}{ Grupo/Participantes } & \multirow{2}{*}{$\begin{array}{c}\text { Valor do Componente } 1 \text { (FR) } \\
\text { ao final da Fase } 2 \text { (LB) }\end{array}$} & \multicolumn{2}{|c|}{$\begin{array}{l}\text { Taxa de pontos por componente } \\
\text { na última sessão da Fase } 2 \text { (LB) }\end{array}$} & \multicolumn{2}{|c|}{$\begin{array}{c}\text { Taxa de pontos por componente } \\
\text { nas Fases } 1 \text { e } 2 \\
\end{array}$} \\
\hline & & FR & DRL & FR & DRL \\
\hline $\mathrm{G} 1 / \mathrm{P} 1$ & 120 & 1,8 & 1,67 & 4,29 & 1,93 \\
\hline $\mathrm{G} 1 / \mathrm{P} 2$ & 110 & 3,07 & 2,07 & 4,01 & 1,65 \\
\hline G1/P3 & 110 & 2,20 & 1,87 & 3,33 & 2,06 \\
\hline G1/P4 & 120 & 2,67 & 2,27 & 5,74 & 2,68 \\
\hline G1/P5 & 100 & 2,67 & 1,73 & 4,58 & 2,16 \\
\hline G2/P6 & 120 & 2,60 & 1,73 & 3,24 & 1,55 \\
\hline $\mathrm{G} 2 / \mathrm{P} 7$ & 120 & 2,13 & 1,60 & 2,66 & 1,69 \\
\hline $\mathrm{G} 2 / \mathrm{P} 10$ & 120 & 2,07 & 2,00 & 2,85 & 1,43 \\
\hline $\mathrm{G} 3 / \mathrm{P} 11$ & 135 & 2,07 & 2,27 & 2,76 & 1,69 \\
\hline $\mathrm{G} 3 / \mathrm{P} 12$ & 100 & 2,00 & 2,20 & 2,44 & 1,51 \\
\hline $\mathrm{G} 3 / \mathrm{P} 15$ & 110 & 2,67 & 2,27 & 2,94 & 1,88 \\
\hline \multirow[t]{2}{*}{$\mathrm{G} 3 / \mathrm{P} 16$} & 110 & 2,60 & 1,80 & 2,93 & 1,55 \\
\hline & $\begin{array}{l}\text { Média } \\
\pm \mathrm{DP}\end{array}$ & $\begin{array}{c}2,38 \\
\pm 0,38\end{array}$ & $\begin{array}{c}1,96 \\
\pm 0,25\end{array}$ & $\begin{array}{c}3,48 \\
\pm 0,98\end{array}$ & $\begin{array}{c}1,81 \\
\pm 0,35\end{array}$ \\
\hline
\end{tabular}




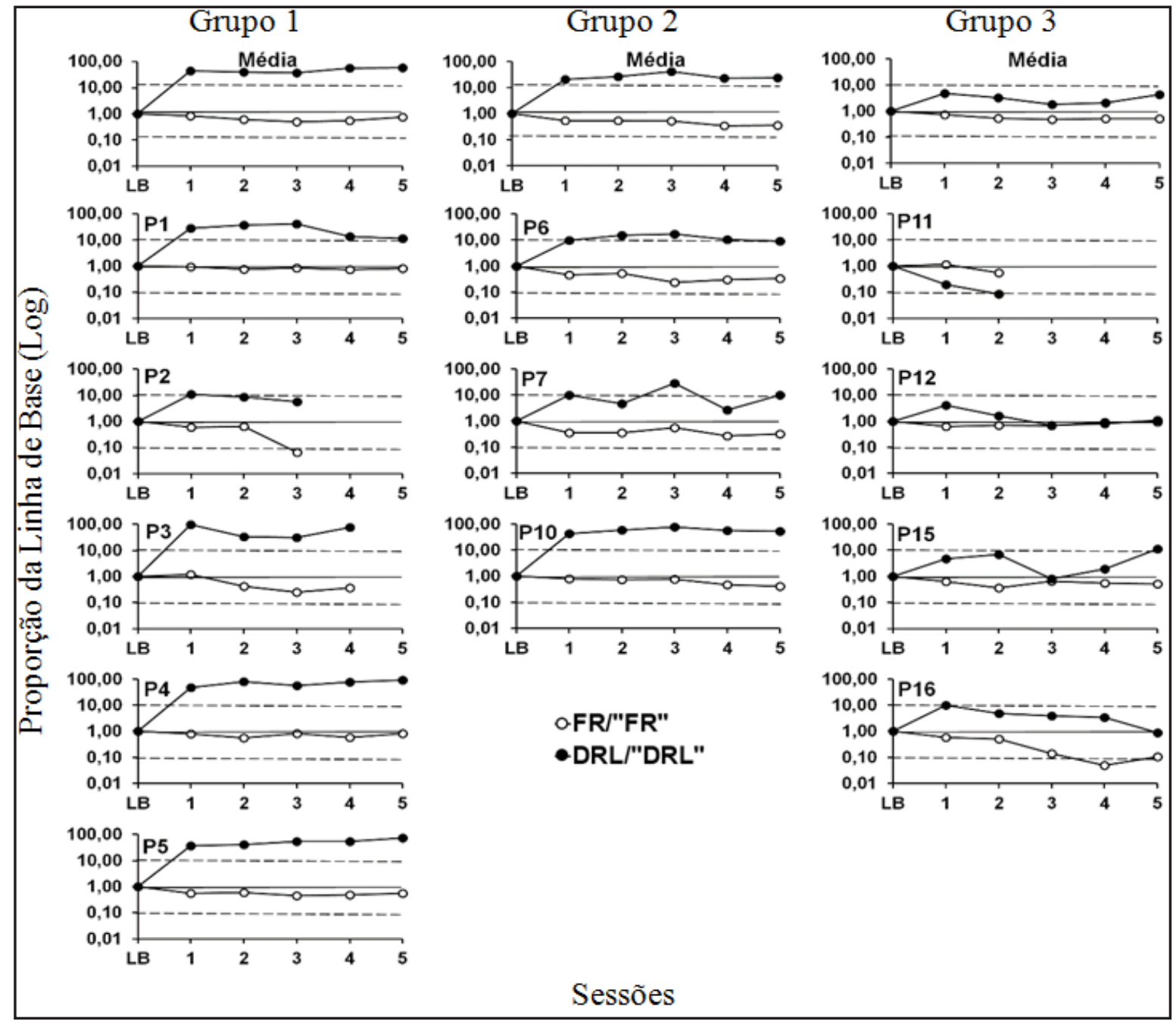

Figura 2. Taxa de respostas no componente "FR" (círculo vazio) e no componente "DRL" (círculo cheio) nas sessões da Fase 3 como uma proporção da taxa de respostas nos componentes FR e DRL, respectivamente, da Linha de Base (LB) da Fase 2. Quanto mais próxima de 1,0, menor a mudança da taxa de respostas em relação à LB. As linhas tracejadas em 10,0 e 0,1 indicam uma mudança de 10 vezes o valor LB, para mais ou para menos, respectivamente. Os gráficos superiores exibem a média dos participantes de cada grupo

participantes dos Grupos 1 e 2, isso não ocorre para dois (P11 e P16) dos quatro participantes do Grupo 3.

Em suma, consistente com os resultados das figuras $1 \mathrm{e}$ 2, a Figura 3 mostra que, de maneira geral, apesar de altas taxas de respostas serem mantidas no início da exposição ao "FR" para os três grupos, mais pausas foram observadas nos Grupos 1 e 2. Por sua vez, a taxa de respostas no "DRL" aumentou, principalmente para os Grupos 1 e 2. Em outras palavras, o responder em "FR" foi mais resistente à mudança do que em "DRL", e instruções aumentaram essa resistência, especialmente no componente previamente relacionado ao DRL.

\section{Discussão}

Tomados em conjunto, os resultados da presente pesquisa parecem indicar que instruções sobre o responder, exposição gradual e exposição direta ao múltiplo FR 60
DRL 20 s selecionam taxa de respostas diferenciadas entre os componentes. Entretanto, com os critérios adotados na presente pesquisa, a instrução (Grupo 3) produziu aquisição diferenciada das taxas de respostas mais rapidamente do que a exposição gradual (Grupo 1) que, por sua vez, produziu aquisição mais rápida do que a exposição direta (Grupo 2). $\mathrm{O}$ comportamento instruído foi mais resistente à mudança (i.e., demorou mais tempo para começar a mudar) quando houve a suspensão do reforço.

\section{Aquisição}

Um dos objetivos da presente pesquisa foi verificar os efeitos do aumento gradual nos valores de um esquema múltiplo FR DRL (Grupo 1) versus a exposição direta a esse esquema (Grupo 2) sobre a diferenciação na taxa de respostas nos componentes do múltiplo. De forma geral, todos os participantes com exposição gradual atingiram os 


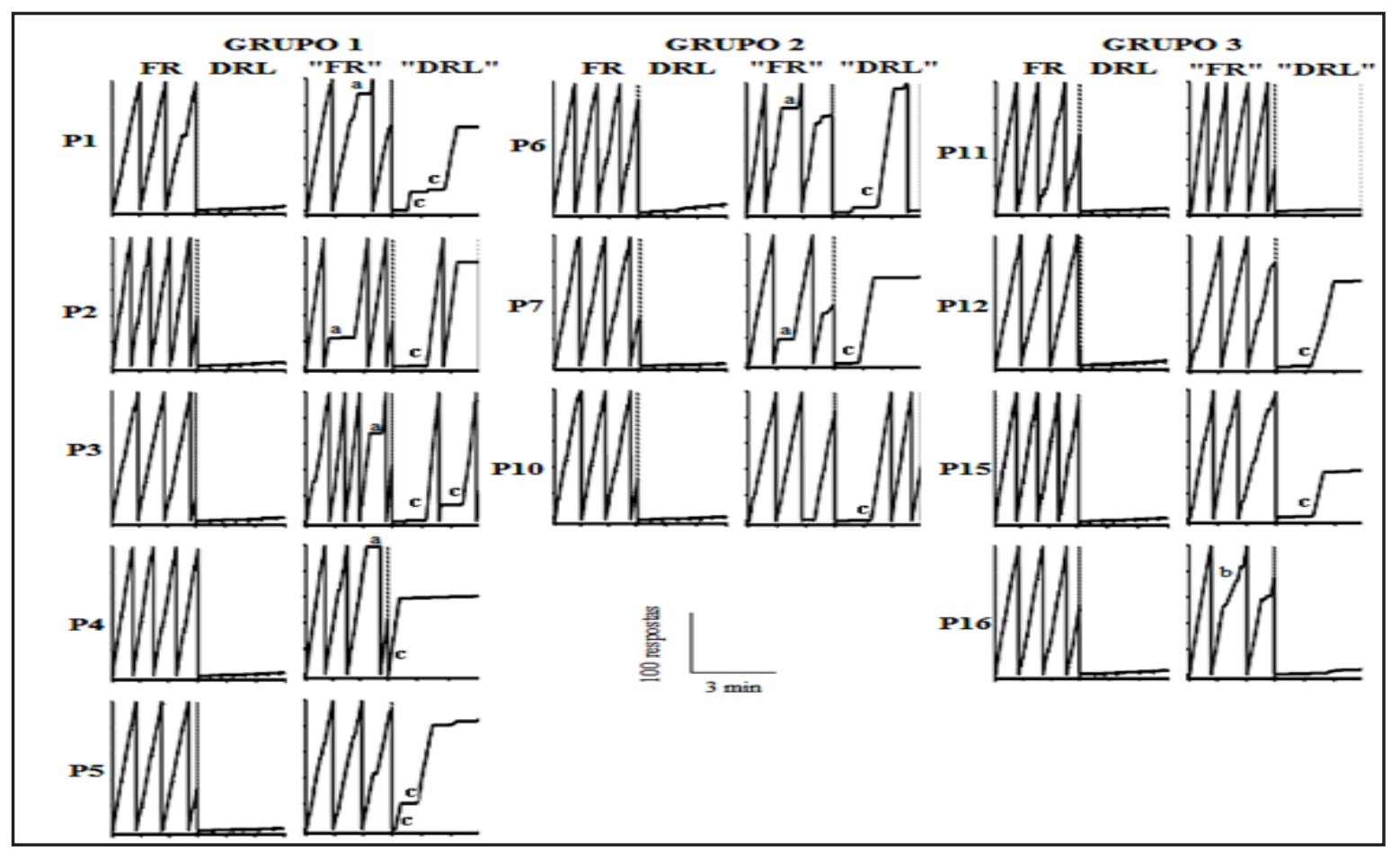

Figura 3. Registro cumulativo de pressão ao botão durante a última apresentação dos componentes FR e DRL (Fase 2) e primeira apresentação dos componentes "FR" "DRL" (Fase 3). A curva de respostas acumuladas retorna azero após a ocorrência de 250 respostas. Marcas diagonais nas curvas indicam o momento em que ocorreu o aparecimento do smile (reforço).

critérios de aquisição do comportamento em três ou quatro sessões, replicando os resultados de estudos em que houve diferenciação de respostas (independentemente do número de sessões requeridas) quando os parâmetros do esquema foram gradualmente aumentados (e.g., Costa, et al., 2012; Okouchi, 1999; Porto, et al., 2011). Dos cinco participantes com exposição direta, três atingiram os critérios de aquisição em até quatro sessões e dois não atingiram esses critérios em quatro sessões.

Esses resultados do Grupo 2 poderiam ir ao encontro da sugestão feita em Porto et al. (2011) de que a exposição direta aos valores finais do múltiplo FR DRL dificulta a diferenciação das taxas de respostas. Entretanto, esses resultados devem ser interpretados com cautela, pois todos os participantes do Grupo 2-Exposição direta emitiram taxas de respostas diferenciadas (com ID acima de 0,5) a partir da terceira sessão da Fase 1 e P18, mesmo tendo sido eliminado do experimento, atingiu valores próximos aos critérios de aquisição (ID=0,89 e \% DRL=42,0).

Os resultados do presente experimento vão ao encontro dos resultados de Hayes, Brownstein, Hass et al. (1986) e de Hayes, Brownstein, Zettle et al. (1986, Experimento 1) no que diz respeito à maior rapidez na aquisição do comportamento instruído. O Grupo 2 teve o menor número de participantes que atingiu os critérios estabelecidos; e mesmo considerando apenas os participantes para os quais os critérios foram atingidos, o Grupo 2 foi aquele em que o maior número de sessões foram necessárias. Esses resultados indicam que o aumento gradual nos parâmetros do esquema (Grupo 1) e a instrução (Grupo 3) sobre como responder facilitaram a aquisição do comportamento em relação à condição onde nenhuma dessas variáveis esteve presente.

De forma geral, os participantes do Grupo 3 atingiram primeiro o critério de ID e, posteriormente, o critério de porcentagem de pontos ganhos em DRL durante a Fase 1-Aquisição. Uma das variáveis que pode ter sido responsável pelo tempo necessário para que o comportamento ficasse sob o controle do DRL - e, então, $50 \%$ dos pontos disponíveis fossem obtidos - foi o tipo de instrução. $O$ trecho da instrução adicionado para os participantes do Grupo 3 discorria sobre a taxa de respostas que produziria o ganho de pontos: "você deverá pressionar (...) devagar quando o botão estiver amarelo", mas não especificava quanto tempo deveria se passar entre uma pressão e outra. Nos estudos que concluíram que a instrução produzia aquisição do comportamento mais rapidamente (e.g., Hayes, Brownstein, Hass et al., 1986; Hayes, Brownstein, Zettle, et al., 1986, Experimento 1) os participantes receberam instruções semelhantes às do presente estudo, entretanto o componente DRL desses estudos era de $6 \mathrm{~s}$. Isto parece indicar que "pressionar devagar" é uma instrução mais provável de ser acurada para um DRL $6 \mathrm{~s}$ do que para um DRL $20 \mathrm{~s}$, por ser um parâmetro menos exigente (aguardar 6 segundos para responder em um computador é mais provável do que aguardar por 20 segundos). Investigações futuras poderiam ser realizadas para 
avaliar o efeito de uma instrução acerca da taxa de respostas (e.g., "pressione rapidamente" ou "pressione lentamente") e uma instrução acerca da contingência programada (e.g., "pressione $\mathrm{n}$ vezes para obter um ponto" ou "aguarde $\mathrm{t}$ segundos para pressionar e então ganhar um ponto") sobre o tempo necessário para que a taxa de respostas fique sob o controle de um múltiplo FR DRL.

\section{Resistência à extinção}

A presente pesquisa teve como objetivo, também, verificar os efeitos de diferentes formas de exposição ao esquema múltiplo FR DRL sobre a resistência à mudança frente à suspensão do reforço. A taxa de respostas no "FR" foi menos alterada do que no "DRL", resultados semelhantes aos de Soares et al. (2013). Esse resultado não corrobora, no entanto, os de Lattal (1989) e Nevin (1974, Experimento 5) que observaram maior resistência à mudança para o comportamento previamente emitido em baixa taxa do que para o comportamento previamente emitido em alta taxa. Todavia, os resultados de Lattal e de Nevin foram obtidos quando a taxa de reforços foi semelhante entre os componentes. No presente estudo, embora a Fase 2 tenha sido planejada, entre outras coisas, com o objetivo de aproximar o ganho de pontos nos dois componentes do múltiplo FR DRL, a taxa de pontos foi diferente quando se considera todas as sessões prévias à extinção (ver última coluna na Tabela 3). Esse problema também foi observado no estudo de Soares et al., não tendo sido contornado no presente trabalho porque os dados já haviam sido coletados quando o problema foi identificado em Soares et al.

O registro cumulativo, exibido na Figura 3, tornou possível a verificação do momento em que aconteceu a alteração do padrão de respostas anteriormente selecionado. Constatou-se alteração no padrão de respostas emitido no "FR" mais rapidamente para os participantes que não receberam instruções (Grupo 1 e 2) do que para os participantes que obtiveram instruções acerca da taxa de respostas (Grupo 3). É importante ressaltar que essa diferença foi sutil e não teve efeitos de longa duração, pois, como observado anteriormente, a análise da proporção de mudança entre sessões para o "FR" (Figura 2) não apresentou diferenças entre grupos.

Em linhas gerais, verificou-se que a exposição direta aos valores finais do múltiplo FR DRL com instrução (Grupo 3) produziu mudança de menor magnitude no "DRL" durante a extinção do que a exposição gradual (Grupo 1) ou exposição direta, sem instrução (Grupo 2), pois para cinco de oito participantes que não receberam instrução (Grupo 1 e 2) e concluíram o estudo, a taxa de respostas no "DRL" atingiu, durante a extinção, valores 10 vezes acima o valor da linha de base, enquanto para nenhum participante do Grupo 3 observou-se tal alteração para este componente (ver Figura 2). Portanto, tomando-se os resultados do "DRL" entre os grupos, o comportamento adquirido por meio da instrução (Grupo 3) produziu taxas de respostas que mudaram menos em relação aos grupos que não tiveram instrução. Esses resultados vão ao encontro dos de Podlesnik e Chase (2006) que também verificaram maior resistência à mudança para o comportamento que foi instruído do que para o comportamento exposto diretamente às contingências, mantidos em um esquema múltiplo de intervalo variável (VI), quando o evento disruptivo foi um vídeo de comédia. Além disso, esses resultados vão ao encontro de outros estudos que mostraram menor sensibilidade comportamental (i.e., maior resistência à mudança) do comportamento instruído, comparado ao comportamento modelado pelas contingências (e.g., Albuquerque et al., 2003; Hayes, Brownstein, Hass et al. 1986; Hayes, Brownstein, Zettle et al., 1986; Rosenfarb et al., 1992).

\section{Referências}

Albuquerque, L. C., de Souza, D. G., Matos, M. A., \& Paracampo, C. C. P. (2003). Análise dos efeitos de histórias experimentais sobre o seguimento subsequente de regras. Acta Comportamentalia, 11(1), 87-126.

Catania, A. C. (1999). Aprendizagem: comportamento, linguagem e cognição (4ª . ed., D. G. de Souza et al., (Trads.). Porto Alegre: Artes Médicas

Cerutti, D. T. (1989).Discrimination theory of rule-governed behavior. Journal of the Experimental Analysis of Behavior, 51(2), 259-276.

Costa, C. E., \& Banaco, R. A. (2002). ProgRef v3: sistema computadorizado para a coleta de dados sobre programas de reforço com humanos - recursos básicos. Revista Brasileira de Terapia Comportamental e Cognitiva, 4, 171-172.

Costa, C. E., \& Banaco, R. A. (2003). ProgRef v3: sistema computadorizado para a coleta de dados sobre programas de reforço com humanos - recursos adicionais. Revista Brasileira de Terapia Comportamental e Cognitiva, 5, 219-229.

Costa, C. E., \& Cançado, C. R. X. (2012). Stability check: A program for calculating the stability of behavior. Mexican Journal of Behavior Analysis, 38(1), 61-71.

Costa, C. E., Soares, P. G., \& Ramos, M. N. (2012). Controle de estímulos e história comportamental: uma replicação sistemática de Freeman e Lattal (1992). Temas em Psicologia da SBP, 20(1), 273-288.

dos Santos, C. V. (2005). Momento comportamental. Em J. AbreuRodrigues \& M. R. Ribeiro (Eds.), Análise do Comportamento: pesquisa, teoria e aplicação (pp. 63-80). Porto Alegre: Artmed.

Hayes, S. C., Brownstein, A. J., Haas, J. R., \& Greenway, D. E. (1986). Instructions, multiple schedules, and extinction: Distinguishing rule-governed from schedule-controlled behavior. Journal of the Experimental Analysis of Behavior, 46(2), 137-147.

Hayes, S. C., Brownstein, A. J., Zettle, R. D., Rosenfarb, I., \& Korn, Z. (1986). Rule-governed behavior and sensitivity to changing consequences of responding. Journal of the Experimental Analysis of Behavior, 45(3), 237-256.

Lattal, K. A. (1989). Contingencies on response rate and resistance to change. Learning and Motivation, 20, 191-203.

Madden, G. J., Chase, P. N., \& Joyce, J. H. (1998). Making sense of sensitivity in the human operant literature. The Behavior Analyst, 21(1), 1-12.

Nevin, J. A. (1974). Response strength in multiple schedules. Journal of the Experimental Analysis of Behavior, 21(3), 389-408. 
Okouchi, H. (1999). Instructions as discriminative stimuli. Journal of the Experimental Analysis of Behavior, 72, 205-214.

Perone, M. (1991). Experimental design in the analysis of freeoperant behavior. Em I. H. Iversen \& K. A. Lattal (Orgs.), Experimental Analysis of Behavior - Part I (pp. 59-85). New York: Elsevier.

Podlesnik, C. A., \& Chase, P. N. (2006). Sensitivity and strength: Effects of instruction on resistance to change. The Psychological Record, 56, 303-320.

Porto, T. H., Ramos, M. N., \& Costa, C. E. (2011). História de aquisição do comportamento em um múltiplo FR-DRL: diferenciação e estabilidade das taxas de respostas. Acta Comportamentalia, 19(3), 281-306.

Rosenfarb, I. S., Newland, M. C., Brannon, S. E., \& Howey, D. S. (1992). Effects of self-generated rules on the development of schedule-controlled behavior. Journal of the Experimental Analysis of Behavior, 58(1), 107-121.
Rumbold, G. R., \& White, J. M. (1987). Effects of repeated alcohol administration on human operant behaviour. Psychopharmacology, 92(2), 186-191.

Schoenfeld, W. N., Cumming, W. W., \& Hearst, E. (1956). On the classification of reinforcement schedules. Proceedings of the National Academy of Sciences of the United States of America, 42, 563-570.

Soares, P. G., Costa, C. E., Cançado, C. R. X., \& Cirino, S. D. (2013). Controle de estímulos e história comportamental em humanos. Psicologia: Reflexão e Crítica, 26(2), 357-366.

Skinner, B. F. (1969). Contingencies of reinforcement: A theoretical analysis. New York: Appleton-Century-Crofts.

Recebido em 01.10.2013

Primeira decisão editorial em 13.01.2014

Versão final em 05.08.2014

Aceito em 11.08.2014 